University of Wollongong

Research Online

Faculty of Engineering - Papers (Archive)

Faculty of Engineering and Information

Sciences

2003

\title{
Magnetic hysteresis and relaxation in Bi2212 single crystals doped with Fe and $\mathrm{Pb}$
}

K. K. Uprety

University of Wollongong

J. Horvat

University of Wollongong, jhorvat@uow.edu.au

Xiaolin Wang

University of Wollongong, xiaolin@uow.edu.au

G. D. Gu

Brookhaven National Laboratory, New York, USA

M. Ionescu

University of Wollongong, mionescu@uow.edu.au

See next page for additional authors

Follow this and additional works at: https://ro.uow.edu.au/engpapers

Part of the Engineering Commons

https://ro.uow.edu.au/engpapers/33

\section{Recommended Citation}

Uprety, K. K.; Horvat, J.; Wang, Xiaolin; Gu, G. D.; Ionescu, M.; Liu, Hua-Kun; Dou, S. X.; and Brandt, E. H.: Magnetic hysteresis and relaxation in Bi2212 single crystals doped with Fe and Pb 2003.

https://ro.uow.edu.au/engpapers/33 


\section{Authors}

K. K. Uprety, J. Horvat, Xiaolin Wang, G. D. Gu, M. Ionescu, Hua-Kun Liu, S. X. Dou, and E. H. Brandt 


\title{
Magnetic Hysteresis and Relaxation in Bi2212 Single Crystals Doped With Fe and Pb
}

\author{
K. K. Uprety, J. Horvat, X. L. Wang, G. D. Gu, M. Ionescu, H. K. Liu, S. X. Dou, and E. H. Brandt
}

\begin{abstract}
Magnetic hysteresis and magnetic relaxation measurements have been performed to study vortex pinning behaviors for pure, $\mathrm{Fe}$ doped and heavily $\mathrm{Pb}$ doped $\mathrm{Bi} 2212$ single crystals. Unlike pure and Fe doped Bi2212 crystals, heavily $\mathrm{Pb}$ doped crystal showed strong vortex pinning behavior. We interpret the strong pinning in heavily $\mathrm{Pb}$ doped $\mathrm{Bi} 2212$ single crystals as arising from the improved Josephson coupling in Bi2212 single crystal after heavy $\mathrm{Pb}$ doping. In heavily $\mathrm{Pb}$ doped single Bi2212 crystals, $H_{\mathrm{dis}}(T)$ was observed to decrease with increasing $T$. Here, $H_{\text {dis }}(T)$ is an order-disorder field that separates a weakly elastically disordered vortex lattice from a plastically disordered vortex solid. However, in pure and Fe doped Bi2212 single crystals, $H_{\text {dis }}(T)$ was observed to be temperature independent. We also report a significant shift of $T_{\mathrm{CR}}$, a crossover temperature separating two pinning regimes, toward higher temperatures with heavy $\mathrm{Pb}$ doping of Bi2212 single crystals. On the other hand $T_{\mathrm{CR}}$ did not shift with $\mathrm{Fe}$ doping of $\mathrm{Bi2212}$ single crystals. It is argued that the temperature dependence of $H_{\mathrm{dis}}(T)$ and the shift of $T_{\mathrm{CR}}$ in heavily $\mathrm{Pb}$ doped $\mathrm{Bi} 2212$ crystals was related to the enhanced $c$-axis conductivity caused by the $\mathrm{Pb}$ situated between the $\mathrm{CuO}_{2}$ layers and imposing a 3D characteristic on the vortex lattice.
\end{abstract}

Index Terms-Bi2212 single crystal, crossover temperature, iron, lead, order-disorder field.

\section{INTRODUCTION}

$\mathbf{I}^{\mathrm{r}}$ $\mathrm{N}$ layered superconductors, the vortices for an applied field parallel to the $c$-axis are described by pancake vortices. For less anisotropic materials, such as $\mathrm{YBa}_{2} \mathrm{Cu}_{3} \mathrm{O}_{7-\delta}$ (Y123), the pancake vortices are coupled through Josephson currents forming 3D vortex lines. However, for the large anisotropic materials, such as $\mathrm{Bi}_{2} \mathrm{Si}_{2} \mathrm{CaCu}_{2} \mathrm{O}_{8+\delta}(\mathrm{Bi} 2212)$, the pancake vortices are decoupled due to thermal energy. The decoupled pancake vortices move via Lorentz forces, producing dissipation. The dissipation is one of the serious problems in bringing these materials into technical applications. However, the dissipation can be reduced by improving the vortex pinning and this can be done by introducing pinning centres or improving the Josephson coupling of the pancake vortices.

In this paper, we study the vortex pinning behavior in Bi2212 single crystals doped with $\mathrm{Pb}$ and $\mathrm{Fe}$. In heavily $\mathrm{Pb}$ doped Bi2212 crystals, it is known that $\mathrm{Pb}$ is between $\mathrm{CuO}_{2}$ planes and thus reduces the anisotropy parameter [1], [2]. Our

Manuscript received August 8, 2002. This work was supported by Australian Research Council, Australia.

K. K. Uprety, J. Horvat, X. L. Wang, M. Ionescu, H. K. Liu, and S. X. Dou are with Institute for Superconducting and Electronic Materials, University of Wollongong, Wollongong, NSW 2522, Australia (e-mail: kku01@uow. edu).

G. D. Gu is with Brookhaven National Laboratory, Upton, NY 11973 USA.

E. H. Brandt is with Max-Planck-Institut für Metallforschung, D-70506

Stuttgart, Germany.

Digital Object Identifier 10.1109/TASC.2003.812544 magnetic hysteresis and magnetic relaxation results showed a strong vortex pinning in heavily $\mathrm{Pb}$ doped $\mathrm{Bi} 2212$ single crystals. The improved pinning in Bi2212 single crystals with heavy $\mathrm{Pb}$ doping opens up a potential technological application of these materials in the industry. Based on our observed experimental results, we have proposed that strong pinning behavior in heavily $\mathrm{Pb}$ doped $\mathrm{Bi} 2212$ single crystals came from the improved Josephson coupling of 2D pancake vortices. We will also show that the vortex pinning in $\mathrm{Bi} 2212$ single crystals is not improved by $\mathrm{Fe}$ doping. In Fe doped $\mathrm{Bi} 2212$ single crystals, Fe substitutes into $\mathrm{CuO}_{2}$ planes and does not change its anisotropy parameter [3].

\section{EXPERIMENTAL PROCEDURES}

The heavily $\mathrm{Pb}$ doped $\mathrm{Bi}_{2-x} \mathrm{~Pb}_{x} \mathrm{Sr}_{2} \mathrm{CaCu}_{2} \mathrm{O}_{8}$ crystal was grown by the self-flux method with the nominal $\mathrm{Pb}$ content $x=0.34$ [4]. The size of the $\mathrm{Pb}$ doped single crystal was $0.71 \times 1.11 \times 0.038 \mathrm{~mm}^{3}$, and the critical temperature $T_{c}$ was $69 \mathrm{~K}$. The critical temperature $T_{c}$ of the crystal was obtained from the magnetic ac susceptibility measurement, with the frequency and amplitude of the excitation field of $117 \mathrm{~Hz}$ and 0.1 Oe, respectively. The Fe doped single crystals were prepared by using the floating zone method and the nominal Fe content in the $\mathrm{Bi}_{2.1} \mathrm{Sr}_{1.9} \mathrm{Ca}_{1.0}\left(\mathrm{Cu}_{1-y} \mathrm{Fe}_{y}\right) \mathrm{O}_{8}$ single crystals was $y=0$ and 0.005 [5]. The sizes of the $y=0$ and 0.005 single crystals were $1.95 \times 2.10 \times 0.116 \mathrm{~mm}^{3}$ and $1.3 \times 2.5 \times 0.15 \mathrm{~mm}^{3}$, respectively. The $y=0$, and 0.005 crystals had $T_{c}=88.5$ and $82.25 \mathrm{~K}$, respectively. Here, $y=0$ is a pure Bi2212 single crystal. Magnetic hysteresis loops and magnetic relaxation measurements were performed using Oxford Instruments Vibrating-Sample Magnetometer (VSM). In all measurements, the field $H$ was applied parallel to the $c$-axis of the crystals.

In the hysteresis loop measurements recorded at different temperatures, the magnetic field was changed at a rate of 20 $\mathrm{Oe} / \mathrm{s}$, and the data were recorded at different temperatures.

During the magnetic relaxation measurements, the crystals were first zero-field-cooled from well above $T_{c}$ and then stabilized at a fixed temperature $T$. A magnetic field $H$ larger than the field used in the relaxation measurements by several times the field of full penetration was applied. The field was then lowered to the measuring field and the magnetic moment as a function of time was measured. The relaxation data were recorded at different fields and temperatures. In the experimental results, the experimental points of the first 100 seconds are not included because of uncertainty in the time that passed between the establishment of the field and the measurement of the first experimental point. The following relation has been used to obtain the normalized relaxation rate $S \equiv d \ln \left|M_{\text {in }}\right| / d \ln t$. 


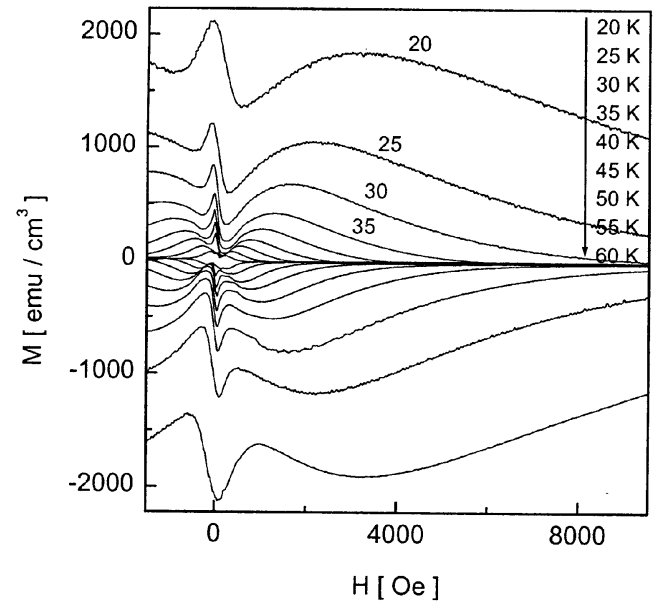

Fig. 1. The magnetic hysteresis loops for a heavily lead doped Bi2212 single crystal at temperatures $T=20,25,30,35,40,45,50,55$, and $60 \mathrm{~K}$.

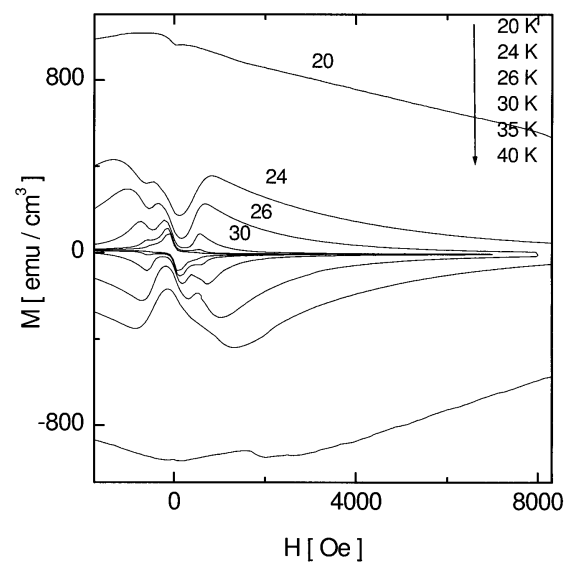

Fig. 2. The magnetic hysteresis curves for pure Bi2212 single crystals at temperatures $T=20,20,24,26,28,33,40 \mathrm{~K}$.

\section{EXPERIMENTAL RESULTS}

Fig. 1 shows the second magnetization peak for heavily $\mathrm{Pb}$ doped Bi2212 single crystal. The peak is seen at all temperatures close to $T_{c}$. However, in pure $(y=0)$ and iron doped $(y=0.005) \mathrm{Bi} 2212$ single crystals, the peak has appeared only between 20 and $40 \mathrm{~K}$ (see Figs. 2 and 3).

Fig. 4 shows $d M / d H$ as a function of the applied field $H$ for pure $(y=0)$ Bi2212 single crystals. The peak in $d M / d H$ has occurred at an inflection point of the second peak in the hysteresis loop at a field $H=H_{\text {infl }}(T)$. We have interpreted $H_{\text {infl }}(T)$ as the order-disorder transition field $H_{\text {dis. }}$. The temperature independence of $H_{\text {infl }}(T)$ was observed for pure and iron doped $\mathrm{Bi} 2212$ single crystals, whereas in heavily $\mathrm{Pb}$ doped single crystals, $H_{\text {inff }}(T)$ was observed to decrease with temperature (see Fig. 5).

The inset to Fig. 4 shows a sharp minimum in $S(H)$ at $H_{\text {inf }}(T)$, indicating two different flux creep processes before and after $H_{\mathrm{inf}}(T)$. The relaxation data and hysteresis loop are also presented along with $S$.

Fig. 6 shows the normalized relaxation rate $S$ as a function of temperature $T$ for pure, Fe doped and heavily $\mathrm{Pb}$ doped Bi2212 single crystals. The peak in $S(T)$ has been proposed as $T_{\mathrm{CR}}$, a

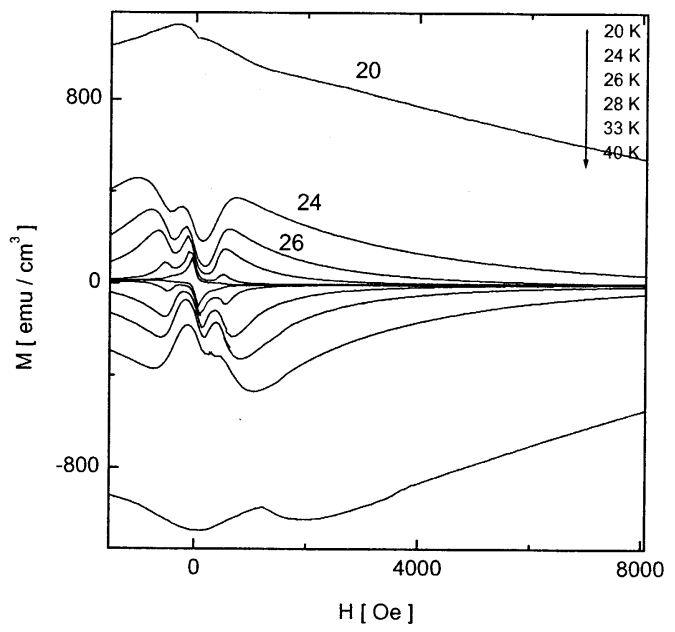

Fig. 3. The magnetic hysteresis curves for $\mathrm{Bi}_{2.1} \mathrm{Sr}_{1.9} \mathrm{Ca}_{1.0}\left(\mathrm{Cu}_{1-y} \mathrm{Fe}_{y}\right)_{2} \mathrm{O}_{8}$ single crystal with Fe concentration $y=0.005$ at temperatures $T=20,20$, $24,26,28,33,40 \mathrm{~K}$.

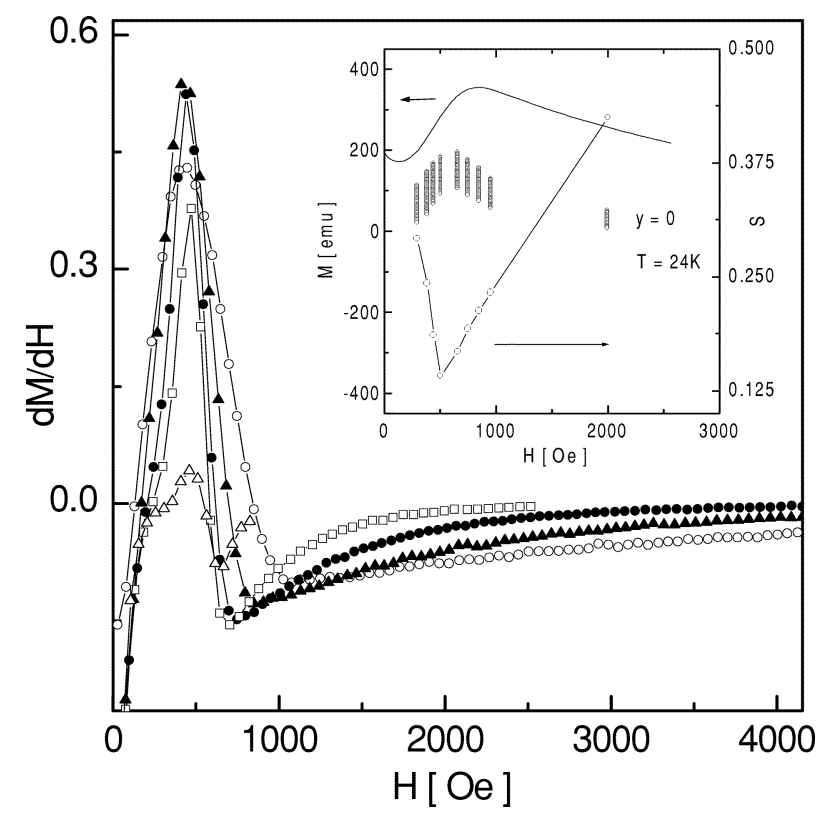

Fig. 4. $\quad d M / d H$ as a function of applied magnetic field $H$ for pure Bi2212 single crystals. The inset shows the magnetization $M$ as a function of applied field $H$ measured at $T=24 \mathrm{~K}$ for pure Bi2212 single crystal. The small circles show the decay of the magnetization $M$ with time $t$ (relaxation between 100 and $3600 \mathrm{sec}$ ) at $T=24 \mathrm{~K}$ for this crystal. The normalized relaxation rate $S$ is indicated by large open circles.

crossover temperature separating two pinning regimes [6], [7]. Fig. 6 shows the peak in $S(T)$ appears at $T=19 \mathrm{~K}$ for pure and Fe doped crystals. However, the peak in $S(T)$ for heavily $\mathrm{Pb}$ doped crystal was observed at $T=35 \mathrm{~K}$ (see inset of Fig. 6).

\section{DISCUSSION}

The strong second magnetization peak, persisting close to $T_{c}$ reflects improved vortex pinning in the heavily $\mathrm{Pb}$ doped Bi2212 crystal, see Fig. 1. A similar strong second peak has been reported in Y123 single crystals close to $T_{c}$ [8]. Pure Bi2212 single crystal has a resistivity anisotropy parameter, $\gamma^{2}=\rho_{c} / \rho_{\mathrm{ab}} \approx 10^{4}-10^{5}$ at $100 \mathrm{~K}$ [1], [9]. Oxygen under-doped Bi2212 single crystal has $\gamma^{2} \approx 10^{5}$, whereas 


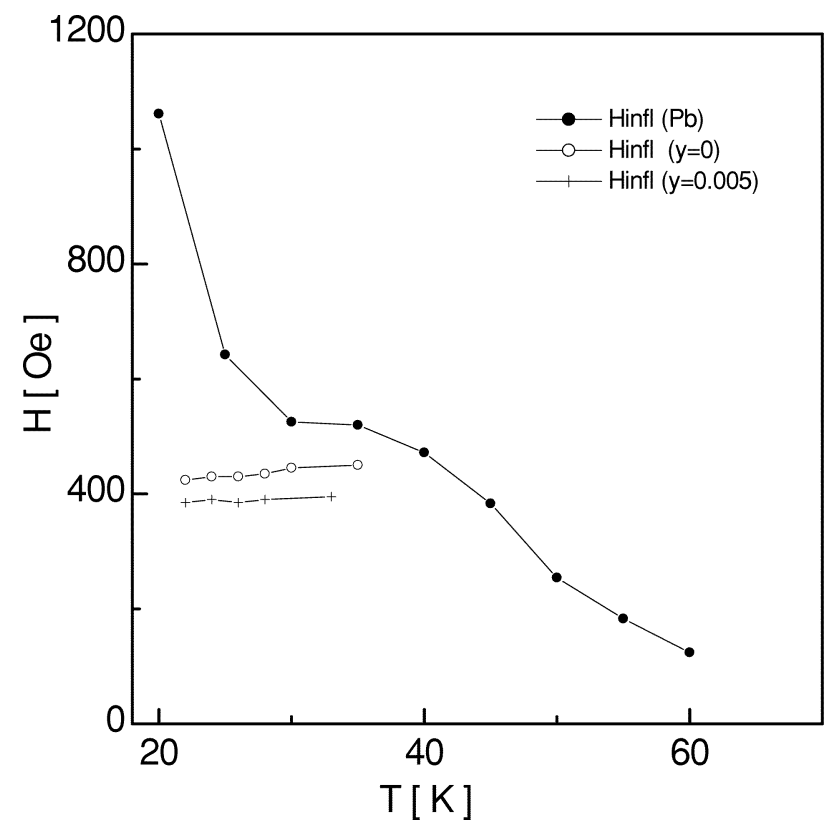

Fig. 5. The order-disorder transition field, $H_{\mathrm{dis}}(T)$ as a function of temperatures for pure, $\mathrm{Fe}$ doped and heavily $\mathrm{Pb}$ doped $\mathrm{Bi} 2212$ single crystals.

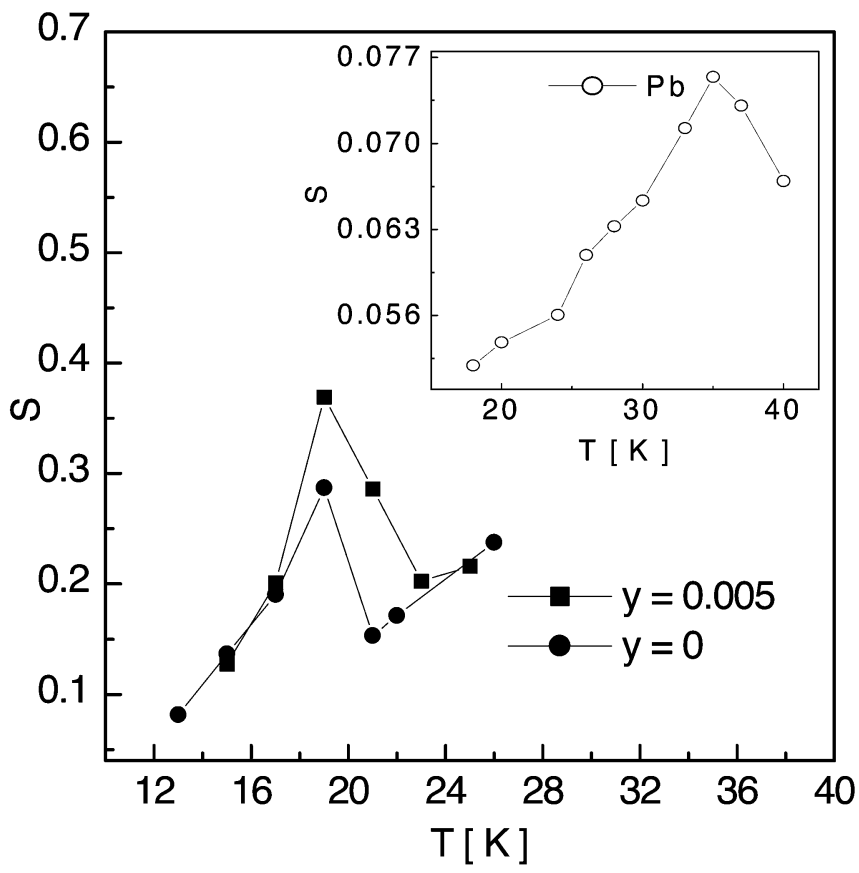

Fig. 6. Normalized relaxation rate vs temperatures for pure, Fe doped and heavily $\mathrm{Pb}$ doped $\mathrm{Bi} 2212$ single crystals. The relaxation are measured at $H=$ 620 Oe for pure Bi2212 single crystal, at $H=520$ Oe for iron doped crystal and at $H=1200$ Oe for heavily $\mathrm{Pb}$ doped Bi2212 single crystal.

oxygen over-doped Bi2212 crystal has $\gamma^{2} \approx 10^{4}$ at $100 \mathrm{~K}$ [9]. However, pure Y123 single crystal has $\gamma^{2} \approx 7-30$ at 100 $\mathrm{K}$ [10]-[12]. This suggested that the strong pinning in Y123 can arise from the strong coupling of 2D pancake vortices. Furthermore, the H-T phase diagram of Y123 shows the presence of 3D vortex line in wide range of fields and temperatures [13]. In heavily $\mathrm{Pb}$ doped single crystal, Motohashi et al. have reported $c$-axis conductivity that was one order of magnitude larger than in pure Bi2212 single crystals, due to a significant reduction of anisotropy in the resistivity $\rho_{c} / \rho_{\mathrm{ab}} \approx 10^{3}$ at $100 \mathrm{~K}$ [1]. Winkeler have reported an increased Josephson interlayer coupling energy by 3.5 times in heavily $\mathrm{Pb}$ doped Bi2212 single crystal, as compared to pure Bi2212 single crystal [14]. The increased coupling energy enhances the layer coupling in heavily $\mathrm{Pb}$ doped single crystal, which thus aligns the pancake vortices into strongly coupled stacks that behave as 3D vortex lines, similar to the vortex lines in Y123. We therefore believe that the strong pinning in the heavily $\mathrm{Pb}$ doped single crystals originates from the enhanced $c$-axis conductivity. Figs. 2 and 3 show that the pinning is not improved in Bi2212 single crystal with $\mathrm{Fe}$ doping. In the $\mathrm{Fe}$ doped single crystals, point defects are reported to reside in the $\mathrm{CuO}_{2}$ planes, since iron replaces $\mathrm{Cu}$ in the plane [3]. The $c$-axis conductivity is not improved with Fe doping in this crystal, and thus pancake coupling does not improve either.

In our previous paper, we have established that $H_{\text {infl }}$, which corresponds to the steepest change of the magnetization (inflection point) on the low field side of the second peak, may be interpreted as the order-disorder transition field, $H_{\mathrm{dis}}$, for pure, Fe doped and heavily $\mathrm{Pb}$ doped Bi2212 single crystals [15]. Here, $H_{\text {dis }}$ separates the weakly disordered vortex lattice from the strongly disordered entangled vortex lattice. In pure Bi2212 single crystals, we observed $H_{\text {dis }} \approx 420$ Oe independent of temperature up to $T=40 \mathrm{~K}$ (see Figs. 4 and 5). A similar result has also been confirmed by Hall probe measurements [16]. The normalized relaxation rate also shows a minimum at $H_{\text {dis }}$ indicating two different creep processes in two different solid regimes (see inset to Fig. 3). In Fe doped Bi2212 single crystals, we observed the temperature independence of $H_{\text {dis }}$. Unlike the pure and $\mathrm{Fe}$ doped Bi2212 single crystals, in heavily $\mathrm{Pb}$ doped Bi2212 single crystals, we have observed temperature dependence of $H_{\text {dis }}$, see Fig. 5. Y123 also has temperature dependence of $H_{\text {dis }}$ [8], [17]. Thus, it is believed that the temperature dependence of $H_{\text {dis }}$ comes from 3D vortex behavior.

We also observed a significant shift of $T_{\mathrm{CR}}$, the crossover temperature that separates two pinning regimes in heavily $\mathrm{Pb}$ doped Bi2212 single crystal (see inset of Fig. 6). A similar shift of $T_{\mathrm{CR}}$ toward higher temperature has been reported for ion irradiation of Bi2212 single crystal [7]. However, for Fe doped crystal, $T_{\mathrm{CR}}$ did not shift toward higher temperature (see Fig. 6). The improved $c$-axis coupling in the ion irradiated Bi2212 crystals has been interpreted as cause of the shift of $T_{\mathrm{CR}}$ toward higher temperature [6], [7]. It is believed that the improved $c$ axis conductivity is responsible for the shift of $T_{\mathrm{CR}}$ in heavily $\mathrm{Pb}$ doped Bi2212 single crystal.

\section{CONCLUSION}

Magnetic hysteresis and magnetic relaxation measurements have been performed in pure, $\mathrm{Fe}$ doped and heavily $\mathrm{Pb}$ doped Bi2212 single crystals. In heavily $\mathrm{Pb}$ doped Bi2212 single crystal, strong second peak in the hysteresis loop up to the critical temperature $T_{c}$ have been observed, whereas in pure and iron doped Bi2212 crystals the peak was observed between 20 and $40 \mathrm{~K}$. We observed a decrease of the order-disorder transition field $H_{\text {dis }}(T)$ with increasing temperature in heavily $\mathrm{Pb}$ doped single crystals. The minimum observed in the field 
dependent normalized relaxation rate $S(H)$ clearly shows two different flux creep processes above and below $H_{\mathrm{dis}}(T)$, indicating two different regimes. In pure and $\mathrm{Fe}$ doped $\mathrm{Bi} 2212$ single crystals, $H_{\mathrm{dis}}(T)$ was found to be field independent. In heavily $\mathrm{Pb}$ doped crystal, we observed a shift of $T_{\mathrm{CR}}$ toward higher temperatures. As for ion irradiated Bi2212 crystal, the enhanced $c$-axis coupling has been implicated in the shift of $T_{\mathrm{CR}}$ with heavy $\mathrm{Pb}$ doping of Bi2212 single crystals [7].

\section{REFERENCES}

[1] T. Motohashi, Y. Nakayama, T. Fujita, K. Kitazawa, J. Shimoyama, and K. Kishio, Phys. Rev. B, vol. 59, p. $14080,1999$.

[2] Z. Hiroi, I. Chong, and M. Takano, J. Solid State Chem, vol. 138, p. 98, 1998.

[3] B. vom Hedt, W. Lisseck, K. Westerholt, and H. Bach, Phys. Rev. B, vol 49, p. 9898, 1994.

[4] J. Horvat, X. L. Wang, and S. X. Dou, Physica C, vol. 324, p. 211, 1999.

[5] G. D. Gu, G. J. Russell, and N. Koshizuka, J. Crystal Growth, vol. 137, p. $472,1994$.
[6] K. K. Uprety, J. Horvat, X. L. Wang, M. Ionescu, H. K. Liu, and S. X. Dou, Supercond Sci and Technol, vol. 14, p. 479, 2001.

[7] V. V. Metlushko, G. Guntherodt, I. N. Goncharov, A. Yu. Didyk, V. V. Moshchalkov, and Y. Bruynseraede, Physica B, vol. 194-196, p. 2219 , 1994.

[8] H. Küpfer, Th. Wolf, C. Lessing, A. A. Zhukov, X. Lançcon, R. MeierHirmer, W. Schauer, and H. Wühl, Phys. Rev. B, vol. 58, p. 2886, 1998.

[9] X. H. Chen, M. Yu, K. Q. Ruan, S. Y. Li, Z. Gui, G. C. Zhang, and L. Z. Cao, Phys. Rev. B, vol. 58, p. 14219, 1998.

[10] G. A. Shams, J. W. Cochrane, and G. J. Russel, Physica C, vol. 324, p. 243, 2001.

[11] S. W. Tozer, A. W. Kleinsasser, T. Denney, D. Kaiser, and F. Holtberg, Phys. Rev. Lett, vol. 59, p. 1768, 1987.

[12] T. A. Friedmann, M. W. Rabin, J. Giapintzakis, J. P. Rice, and D. M. Ginsberg, Phys. Rev. B, vol. 42, p. 6217, 1990.

[13] G. Blatter, M. V. Feigel'man, V. B. Geshkenbein, A. I. Larkin, and V. M. Vinokur, Rev. Mod. Phys, vol. 66, p. 1125, 1994.

[14] L. Winkeler, S. Sadewasser, B. Beschoten, H. Frank, F. Nouvertné, and G. Güntherrodt, Physica C, vol. 265, p. 194, 1996.

[15] K. K. Uprety, J. Horvat, X. L. Wang, M. Ionescu, H. K. Liu, S. X. Dou, and E. H. Brandt, Phys. Rev. B, vol. 65, p. 224 501, 2002.

[16] B. Khaykovich, E. Zeldov, D. Majer, T. W. Li, P. H. Kes, and M. Konczykowski, Phys. Rev. Lett, vol. 76, p. 2555, 1996.

[17] G. M. Mikitik and E. H. Brandt, Phys. Rev. B, vol. 64, p. 184 514, 2001 